\title{
Measuring space radiation shielding effectiveness
}

\author{
Amir Bahadori ${ }^{1,2, \star}$, Edward Semones ${ }^{2}$, Michael Ewert ${ }^{3}$, James Broyan $^{3}$, and Steven Walker ${ }^{4}$ \\ ${ }^{1}$ Kansas State University, Department of Mechanical and Nuclear Engineering, Manhattan, KS, USA 66506 (current address) \\ ${ }^{2}$ NASA Johnson Space Center, Space Radiation Analysis Group, Houston, TX, USA 77058 \\ ${ }^{3}$ NASA Johnson Space Center, Crew \& Thermal Systems Division, Houston, TX, USA 77058 \\ ${ }^{4}$ Old Dominion University, Department of Mathematics \& Statistics, Norfolk, VA, USA 23529
}

\begin{abstract}
Passive radiation shielding is one strategy to mitigate the problem of space radiation exposure. While space vehicles are constructed largely of aluminum, polyethylene has been demonstrated to have superior shielding characteristics for both galactic cosmic rays and solar particle events due to the high hydrogen content. A method to calculate the shielding effectiveness of a material relative to reference material from Bragg peak measurements performed using energetic heavy charged particles is described. Using accelerated alpha particles at the National Aeronautics and Space Administration Space Radiation Laboratory at Brookhaven National Laboratory, the method is applied to sample tiles from the Heat Melt Compactor, which were created by melting material from a simulated astronaut waste stream, consisting of materials such as trash and unconsumed food. The shielding effectiveness calculated from measurements of the Heat Melt Compactor sample tiles is about $10 \%$ less than the shielding effectiveness of polyethylene. Shielding material produced from the astronaut waste stream in the form of Heat Melt Compactor tiles is therefore found to be an attractive solution for protection against space radiation.
\end{abstract}

\section{Introduction}

Human protection from space radiation is a challenging problem that must be addressed using various strategies, including passive shielding, active shielding, improved environment prediction, operational planning and actions, biological uncertainty reduction, and biological countermeasures. In the past, passive shielding studies have focused on using additional mass for the primary purpose of space radiation protection $[1,2]$. However, adding mass for no purpose other than radiation shielding is unpalatable due to the limited launch capability of rockets and the mass required to sustain astronauts for extended periods of time for missions with destinations outside of low-Earth orbit. Therefore, alternative passive shielding strategies, such as using advanced structural materials [3], food [4], or astronaut waste [5] have been suggested. While computational and experimental methods to determine water equivalent thickness for use in proton therapy calculations have been published [6], no standardized procedure has been developed to determine the effectiveness of composite materials for space radiation shielding using measurements without detailed knowledge of the constituents. The purpose of the present study is to develop such a procedure, apply the procedure to sample tiles from the NASA Heat Melt Compactor, and determine whether the Heat Melt Compactor tiles are a viable alternative to dedicated passive shielding constructed from polyethylene.

\footnotetext{
^e-mail: bahadori@ksu.edu
}

\section{Problem Description}

The space radiation environment is comprised of radiation from three sources: geomagnetically-trapped particles, galactic cosmic rays, and solar particle events. Each of these sources presents unique challenges from the perspective of space radiation protection, including the efficacy of passive shielding as a means by which to mitigate space radiation exposure. Since mass launch capability is limited for missions beyond Earth orbit, compacted astronaut waste is attractive as an option for passive shielding against space radiation. No additional launch mass would be required to implement such a shielding strategy. However, the shielding effectiveness of the astronaut waste must be characterized to understand the level of protection afforded by the material. The hypothesis to be tested in the present study is that the Heat Melt Compactor tiles have a shielding effectiveness similar to that of polyethylene due to the high content of plastic and other hydrocarbons in the astronaut waste stream.

\subsection{Space Radiation Environment}

For missions beyond Earth orbit, the contribution of geomagnetically-trapped particles to overall mission radiation exposure can be minimized through judicious selection of trajectories that avoid high intensity regions of Earth's radiation belts. NASA's experience to date with crewed beyond Earth orbit missions consists of the Apollo 


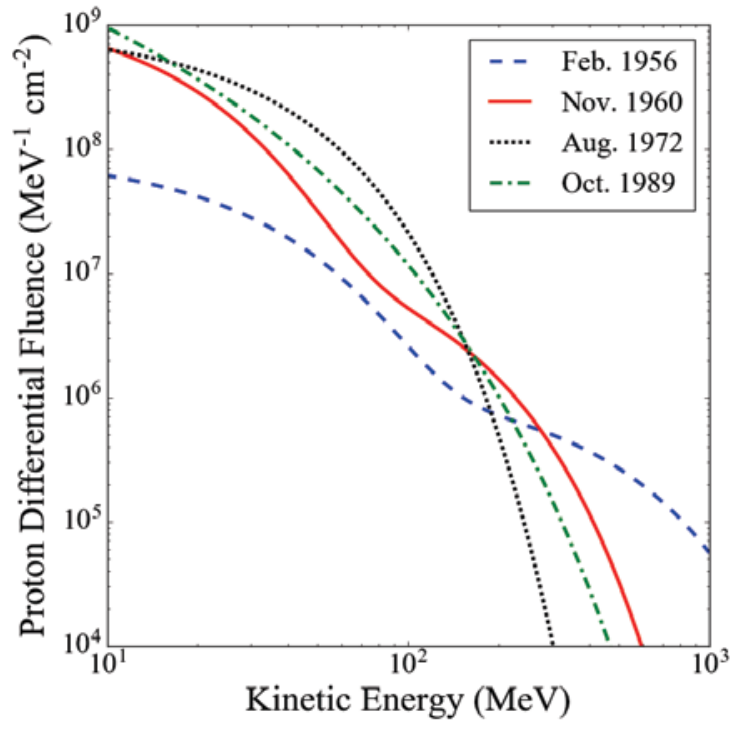

Figure 1. Selected historical solar particle event fluence spectra (reproduced from [9])

program, in which Earth orbits at low altitudes were completed prior to trans-lunar injection [7]. The radiation exposure from geomagnetically-trapped sources was largely limited to fast transits through the particle belts.

Galactic cosmic rays consist of heavy charged particles, ranging in atomic number from 1 (protons) to 58 (nickel ions) and above, with protons comprising about $90 \%$ and alphas comprising about $9 \%$ of the galactic cosmic ray spectrum [8]. The kinetic energies range from fractions of one megaelectron-volt per nucleon $\left(\mathrm{MeV} \mathrm{n}^{-1}\right)$ to thousands of gigaelectron-volts per nucleon $\left(\mathrm{GeV} \mathrm{n}^{-1}\right)$ [8], although the vast majority of ions have kinetic energies less than $50 \mathrm{GeV} \mathrm{n}^{-1}$. The flux density spectra for galactic cosmic ray ions peak at kinetic energies between $100 \mathrm{MeV} \mathrm{n}^{-1}$ and $1 \mathrm{GeV} \mathrm{n}^{-1}$.

Solar particle events are a concern for human space flight in the low cutoff rigidity regions of low-Earth orbit (e.g., near the geomagnetic poles) and outside of lowEarth orbit, such as missions to the Moon or Mars. Several major solar particle events have been observed in the Space Age via balloon measurements or satellites. Several historic solar particle event fluence spectra [9] are shown in Figure 1. These major solar particle events have spectral characteristics that can vary substantially. For example, the February 1956 solar particle event had a substantially larger high energy component than the August 1972 solar particle event [10]. The fluence spectrum impacts the radiation transport and ultimately the exposure and resulting radiation risk to humans within a vehicle or habitat $[11,12]$.

\subsection{Space Radiation Shielding}

Reducing exposure time, increasing distance from the source, and increasing shielding are frequently cited as the three major ways by which radiation exposure levels may be reduced. In space, reducing exposure time is only possible by completing missions with shorter durations or developing faster transit capabilities. Increasing distance from the source is not meaningful since the sources are nearly isotropic. Therefore, shielding remains the only option for reducing space radiation exposure for a given mission.

Galactic cosmic rays are difficult to shield due to the high kinetic energies of the particles and the tendency for ions of higher atomic number to fragment, creating lighter, secondary charged particles. The stopping power of passive shielding is smaller for the lighter ion fragments, which are created with velocities similar to the incident ions, allowing the secondary charged particles to more readily penetrate the shielding. Solar particle events tend to be easier to shield than galactic cosmic rays, as they are comprised mostly of protons with fluence distributions weighted towards lower kinetic energies. However, solar particle events are not easily predicted and can be very intense, resulting in high absolute exposure levels. For both galactic cosmic rays and solar particle events, polyethylene has been shown to have superior shielding characteristics to aluminum [13, 14], which is the dominant constituent in most space vehicle structures. Unfortunately, due to mass constraints for missions beyond Earth orbit, there are no plans to include material for the sole purpose of radiation shielding. Therefore, a desirable passive shielding strategy is to use material required for other purposes as radiation shielding.

\subsection{NASA Heat Melt Compactor}

The NASA Heat Melt Compactor technology has been under development for several years with the goal of developing a device that compacts trash by a factor of seven or more, recovers water from the trash, and produces a stable tile suitable for radiation shielding. Ground testing has demonstrated these capabilities. Plastic in the astronaut waste stream, primarily from food packaging, is melted in the Heat Melt Compactor process and helps encapsulate other items in the trash. The Heat Melt Compactor heats as it compresses in order to sterilize and limit odor as well as to vaporize water for subsequent condensation and recovery.

The first generation Heat Melt Compactor unit at NASA Ames Research Center was used to produce five round tiles for this evaluation with approximate diameter of $20.3 \mathrm{~cm}$ and thickness of $1.9 \mathrm{~cm}$. Figure 2 shows one of the round tiles tested in the present study. The composition of the tiles was estimated based on the constituents of simulated astronaut trash that was processed, which included items such as food packaging materials, wasted food, clothing, and disposable wipes. The second generation Heat Melt Compactor ground unit, which is now being tested, produces square tiles with $22.9 \mathrm{~cm}$ side length. 


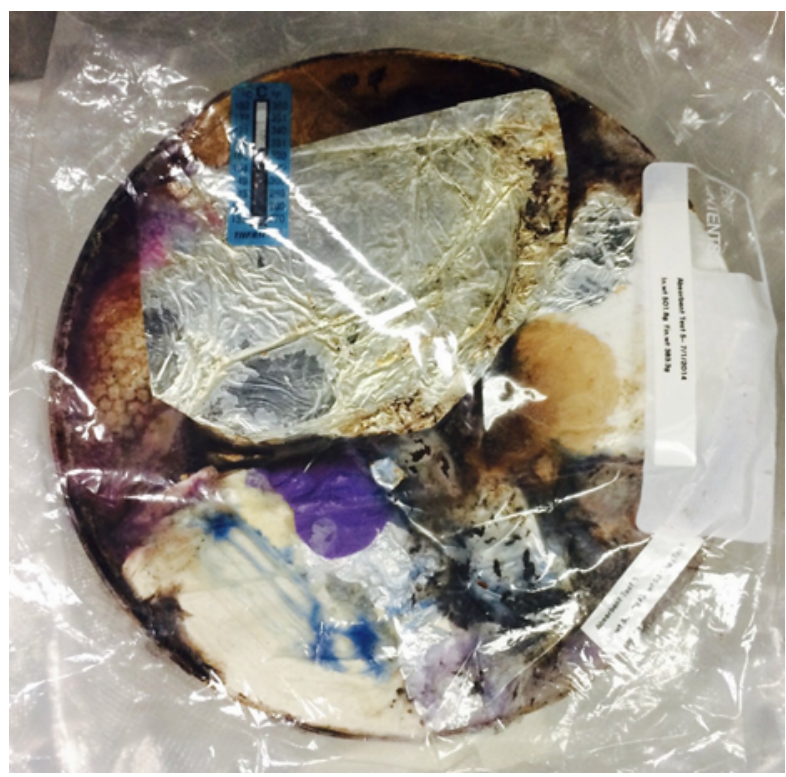

Figure 2. Photograph of Heat Melt Compactor tile tested at NSRL

\section{Methods and Materials}

First, the relative shielding effectiveness of the materialunder-test with respect to the reference material must be defined in terms of measurable or known quantities of both materials using an accelerated charged particle beam. A measurement procedure is then developed to acquire the data necessary to calculate the relative shielding effectiveness.

\subsection{Relative Shielding Effectiveness Definition}

Previously, Guetersloh et al. [15] defined a shielding effectiveness by the ratio of measured absorbed doses with and without shielding material present in the beam. While this is a meaningful definition for a space radiation environment with a broad energy spectrum, it is difficult to interpret when used with a monoenergetic ion beam delivered at an accelerator facility. For certain combinations of ion, energy, and shielding, the measured absorbed dose increases with increasing shielding thickness because of particles slowing within the shield. Therefore, a new definition of shielding effectiveness, defined in terms of a reference material and applicable to accelerator beam measurements, is proposed.

The relative shielding effectiveness $f$ is defined as the ratio of the mass stopping powers for the material-undertest (MUT) and the reference material (RM), as shown in Equation 1. Note that implicit dependence on the particle type and energy is suppressed in this formulation.

$$
f=\frac{\left[-\frac{1}{\rho}\left(\frac{d E}{d x}\right)\right]_{M U T}}{\left[-\frac{1}{\rho}\left(\frac{d E}{d x}\right)\right]_{R M}}
$$

Now, it is assumed that the charged particles have negligible energy loss while traversing the material-under-test sample and the equivalent thickness of reference material, such that the linear stopping power may be calculated as the change in energy resulting from travel through the thickness of each material. Furthermore, the changes in energy for the charged particles traversing each material must be equal. Therefore, Equation 1 reduces to

$$
f=\frac{t_{R M} \rho_{R M}}{t_{M U T} \rho_{M U T}}
$$

where $t_{R M}$ is the equivalent reference material thickness for the material-under-test sample, $\rho_{R M}$ is the density of the reference material, $t_{M U T}$ is the thickness of the material-under-test, and $\rho_{M U T}$ is the density of the material-under-test.

Finally, to account for density and thickness variations in the material-under-test, the areal density of the materialunder-test, which is equal to the denominator of Equation 2 , is simplified to the quotient of the material-under-test sample mass and cross-sectional area. The the relative shielding effectiveness calculation further simplifies to

$$
f=\frac{t_{R M} \rho_{R M}}{\frac{m_{M U T}}{A_{M U T}}}
$$

where $m_{M U T}$ is the mass of the material-under-test sample and $A_{M U T}$ is the cross-sectional area of the material-undertest sample. All values in Equation 3 required for calculation of the relative shielding effectiveness can be measured or are known prior to experiment.

\subsection{Measurement Procedure}

Four quantities must be measured or known to calculate the relative shielding effectiveness for a given materialunder-test sample: the equivalent reference material thickness of the material-under-test sample, the density of the reference material, the mass of the material-under-test sample, and the cross-sectional area of the material-undertest sample.

The equivalent reference material thickness of the material-under-test sample must be determined using experimental measurements at a charged particle accelerator beam line. First, a Bragg peak measurement is performed using reference material to stop the charged particle beam in the detector. The thickness of reference material required is recorded. Next, a Bragg peak measurement is performed with the material-under-test sample proximal to the charged particle beam outlet, and the difference in reference material thickness between the Bragg peak measurement with no material-under-test sample and the Bragg peak measurement with the material-under-test sample is calculated.

The density of the reference material is either known from experience or can be measured by calculating the quotient of the mass and volume. The mass of the material-under-test sample is measured, and the crosssectional area of the material-under-test sample is calculated using measurements of the sample dimensions. It is important that the beam area and detector area envelop the material-under-test sample cross-sectional area to accurately characterize the entire material-under-test sample. 


\section{Data Collection}

For the present study, equivalent reference material thickness data were collected at the NASA Space Radiation Laboratory (NSRL) at Brookhaven National Laboratory in Upton, NY, USA. The ions available for measurement were limited to alphas and ${ }^{56} \mathrm{Fe}$ ions due to scheduling constraints. Measurements using alphas were determined to be most appropriate, as alphas are less likely to fragment than ${ }^{56} \mathrm{Fe}$ ions. Two considerations in addition to applicability to the space radiation environment dictated the selection of the ion kinetic energy. First, the ion kinetic energy must be high enough such that the change in kinetic energy over the Heat Melt Compactor tile is small; and second, the ion kinetic energy must be low enough to permit Bragg peak measurements with the available amount of degrader material. Since measurements for the present study were made in concert with measurements for other projects, a limited selection of kinetic energies was available. Of these, the nominal kinetic energy of $200 \mathrm{MeV} \mathrm{n}^{-1}$ was chosen. Polyethylene is used for performing Bragg peak measurements at the NSRL and therefore served as the reference material for this experiment.

Bragg peak measurements made using only the polyethylene degrader indicated that the alphas had a nominal range of $26.875 \mathrm{~cm}$, corresponding to a kinetic energy of $207.8 \mathrm{MeV} \mathrm{n}^{-1}$. Bragg peak measurements were next performed with each of the five Heat Melt Compactor samples (set-up shown in Figure 3), and the equivalent polyethylene thickness, $t_{P E}$, was found. Here, it was estimated that the Bragg peak measurements have an uncertainty of $0.1 \mathrm{~cm}$. The equivalent polyethylene thicknesses and Heat Melt Compactor sample masses, $m$, are shown in Table 1. The area of each Heat Melt Compactor sample was calculated using a measured diameter of $20.0 \mathrm{~cm}$, yielding a cross-sectional area of $314.2 \mathrm{~cm}^{2}$. The density of the polyethylene used at the NSRL is $0.97 \mathrm{~g} \mathrm{~cm}^{-3}$ [16].

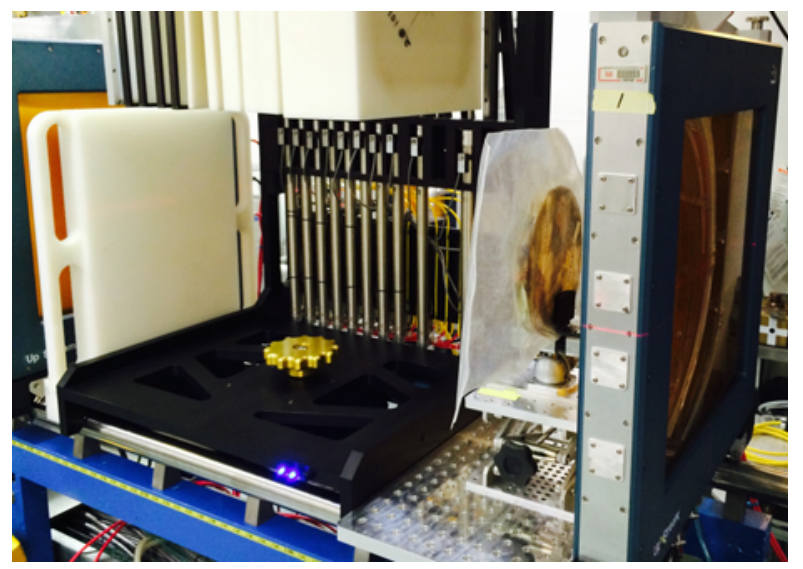

Figure 3. Heat Melt Compactor sample (circular disk in plastic bag) on beam line

\section{Analysis}

The relative shielding effectiveness for each Heat Melt Compactor tile as formulated in Equation 3 was calculated
Table 1. Heat Melt Compactor sample equivalent polyethylene thicknesses and masses

\begin{tabular}{lll}
\hline Tile No. & $t_{P E}(\mathrm{~cm})$ & $m(\mathrm{~kg})$ \\
\hline 1 & $1.2 \pm 0.14$ & 0.398 \\
2 & $1.3 \pm 0.14$ & 0.438 \\
3 & $1.4 \pm 0.14$ & 0.450 \\
4 & $1.3 \pm 0.14$ & 0.435 \\
5 & $1.3 \pm 0.14$ & 0.447 \\
\hline
\end{tabular}

and the results are shown in Table 2. Also shown is the estimated uncertainty for the relative shielding effectiveness. The mean relative shielding effectiveness for the group of samples was found to be $0.91 \pm 0.044$, indicating that the Heat Melt Compactor samples are $91 \% \pm 4.4 \%$ as effective as polyethylene at shielding against alpha particles with a kinetic energy of $207.8 \mathrm{MeV} \mathrm{n}^{-1}$.

Table 2. Heat Melt Compactor sample relative shielding effectiveness experimental values

\begin{tabular}{ll}
\hline Tile No. & $f$ (unitless) \\
\hline 1 & $0.92 \pm 0.11$ \\
2 & $0.91 \pm 0.095$ \\
3 & $0.95 \pm 0.095$ \\
4 & $0.91 \pm 0.098$ \\
5 & $0.89 \pm 0.096$ \\
\hline
\end{tabular}

To check the accuracy of the measurements, the Heat Melt Compactor sample mass stopping power for alpha particles with kinetic energy of $831.2 \mathrm{MeV}$, corresponding to the beam kinetic energy of $207.8 \mathrm{MeV} \mathrm{n}^{-1}$, was calculated using SRIM-2013 [17] for the assumed composition shown in Table 3. The Heat Melt Compactor sample mass stopping power was found to be $0.01700 \mathrm{MeV} \mathrm{cm}^{2} \mathrm{mg}^{-1}$, while the polyethylene mass stopping power was found to be $0.01864 \mathrm{MeV} \mathrm{cm}^{2} \mathrm{mg}^{-1}$. The results from SRIM-2013 indicate a material with the assumed composition is $91.2 \%$ as effective as polyethylene at shielding against alpha particles with kinetic energy of $207.8 \mathrm{MeV} \mathrm{n}^{-1}$, exhibiting excellent agreement with the experimentally-determined relative shielding effectiveness.

Table 3. Heat Melt Compactor sample assumed composition

\begin{tabular}{ll}
\hline Element & Mass fraction \\
\hline $\mathrm{H}$ & 0.08 \\
$\mathrm{C}$ & 0.54 \\
$\mathrm{~N}$ & 0.01 \\
$\mathrm{O}$ & 0.24 \\
$\mathrm{~F}$ & 0.04 \\
$\mathrm{Na}$ & 0.01 \\
$\mathrm{Al}$ & 0.05 \\
$\mathrm{Cl}$ & 0.02 \\
$\mathrm{Fe}$ & 0.01 \\
\hline
\end{tabular}

Both galactic cosmic ray and solar particle event environments are dominated by protons as previously described. While the measurements performed in the present study used alpha particles, the relative shielding effective- 
ness results are applicable protons of the same velocity (i.e., protons with kinetic energy of $207.8 \mathrm{MeV}$ ).

The ratios of the mass stopping powers for protons and alphas were calculated for the Heat Melt Compactor material and polyethylene using SRIM-2013 to demonstrate applicability to protons. These ratios are shown in Figure 4 as a function of kinetic energy per nucleon. Excellent agreement is observed for the ratios between $2 \mathrm{MeV}$ $\mathrm{n}^{-1}$ and $10 \mathrm{GeV} \mathrm{n}^{-1}$. Additionally, the ratios of the mass stopping powers increase with kinetic energy per nucleon, indicating that the shielding effectiveness of the Heat Melt Compactor material with respect to polyethylene would be even more favorable for ions with greater kinetic energy than that tested in the present study.

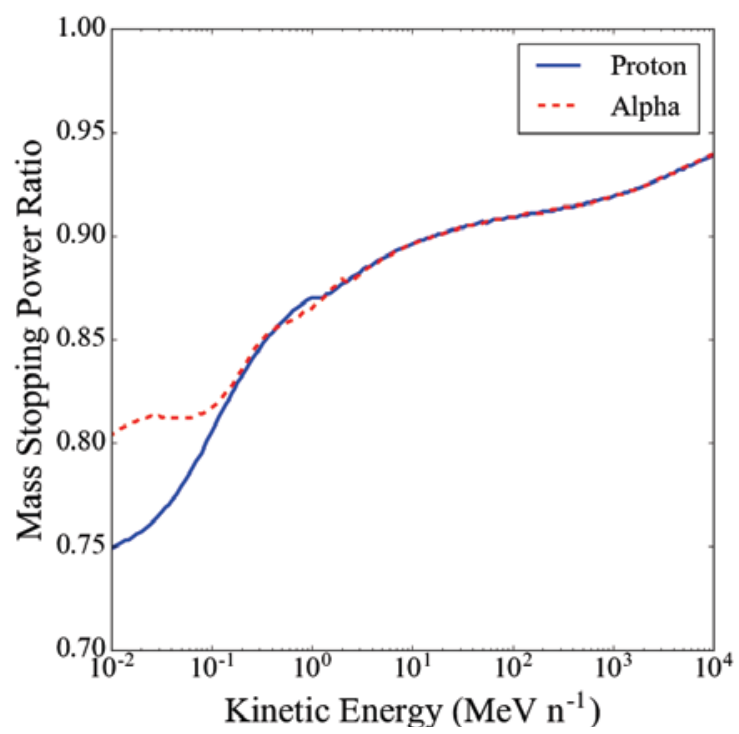

Figure 4. Ratios of Heat Melt Compactor material to polyethylene mass stopping powers for protons and alphas

Simulations of the August 1972 solar particle event using the King model [10] and the 1977 solar minimum galactic cosmic ray environment [18] have been performed using the assumed composition for the Heat Melt Compactor samples. The NASA On-Line Tool for the Assessment of Radiation In Space [19] was used to simulate the effective dose using the FAX female astronaut computational phantom [20] shielded by Heat Melt Compactor material in a spherical shell configuration for shield thicknesses between 0 and $100 \mathrm{~g} \mathrm{~cm}^{-2}$. It is noted that the effective dose calculation uses both the linear energy transferdependent quality factor and tissue weighting factors defined in ICRP Publication 60 [21] and NCRP Report No. 132 [22] without gender averaging. Simulations were also performed for aluminum and polyethylene shielding for comparison.

The results for the August 1972 solar particle event and the 1977 solar minimum galactic cosmic ray spectrum are shown in Figures 5 and 6, respectively. The simulation results for both environments indicate that the performance of the Heat Melt Compactor material is superior to alu- minum and similar in trend, although inferior, to polyethylene.

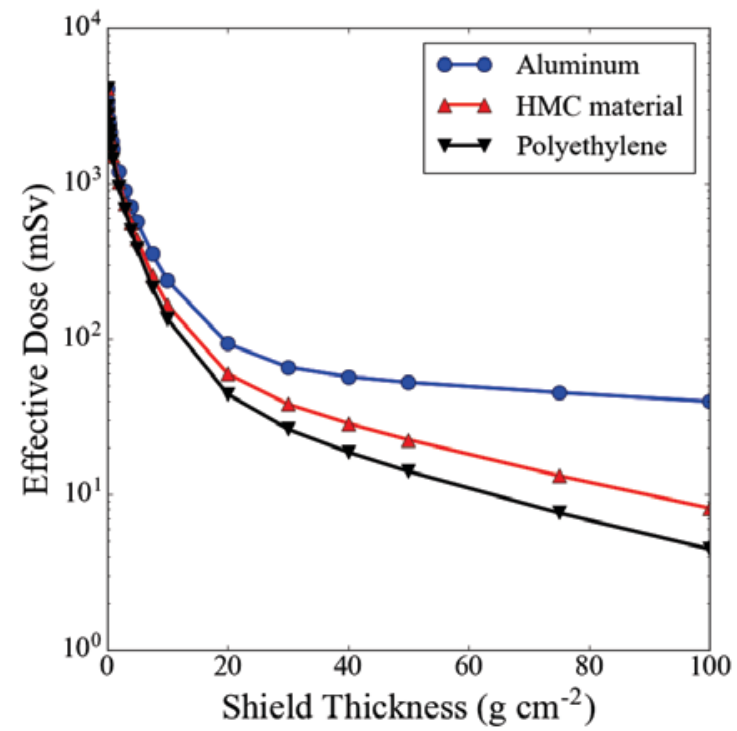

Figure 5. Female effective dose for August 1972 solar particle event environment

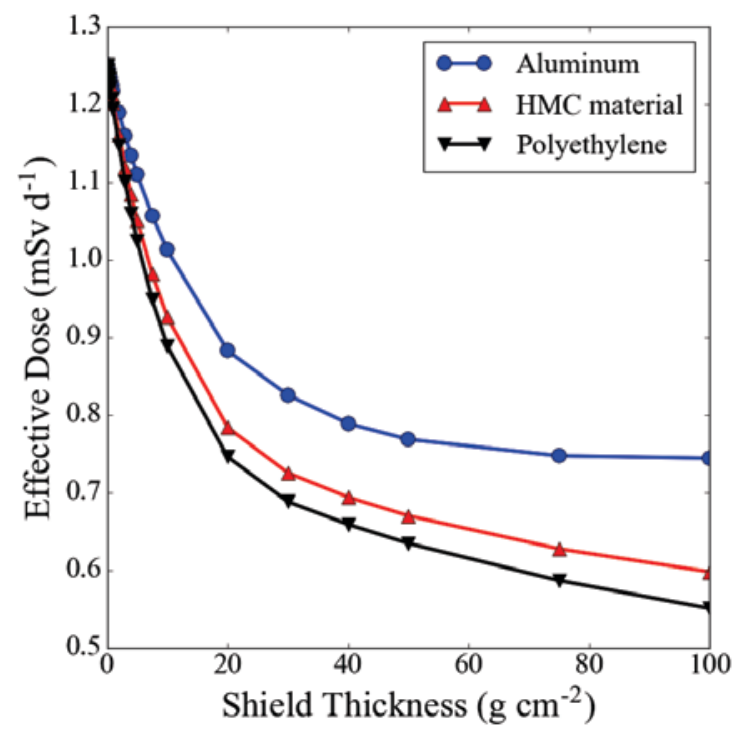

Figure 6. Female effective dose for 1977 solar minimum galactic cosmic ray environment

\section{Conclusions}

The results of the present study indicate that the Heat Melt Compactor material could be used as an acceptable replacement for polyethylene shielding. Simulations of space radiation spectra show that a material with the assumed composition exhibits favorable shielding characteristics. The Heat Melt Compactor material is particu- 
larly attractive since it requires no dedicated mass for radiation shielding. The hypothesis was therefore proven correct, since the space radiation shielding characteristics were shown to be similar to polyethylene.

There are several opportunities for future work investigating the use of the Heat Melt Compactor tiles for space radiation shielding. First, there is the problem of how to incorporate the tiles into the vehicle shielding. They are rigid and not easily conformed to the hull of a spacecraft. Also, there must be some method of tethering them in a particular location. Optimization of placement, including whether they placed externally or internally with respect to the vehicular structural shielding, should be performed. Next, there is a time dependence in the availability of astronaut waste for use in the Heat Melt Compactor. At the beginning of a mission, other consumable materials, such as water or food, that have the reverse time-dependent pattern of availability, should be included in the concept of operations for radiation shielding to make up for the lack of astronaut waste if augmented shielding is required early in a mission of extended duration. Finally, to avoid selection bias and to more completely quantify the shielding effectiveness against solar particle events, simulations should be performed using a proper sample of the historical database of solar particle events.

One limitation of the present study is that neutron contributions are not explicitly considered. Supplementing the measurements with neutron spectrum measurements behind equivalent thicknesses of Heat Melt Compactor material and polyethylene would indicate the degree to which neutron production is enhanced or suppressed in the Heat Melt Compactor material. Another important limitation of the proposed method is that the maximum kinetic energy that can be tested is limited by the amount of reference material available for Bragg peak measurements. Additionally, the kinetic energy must be high enough such that the change in energy experienced by the charged particles traversing the material-under-test sample is small. It is also difficult to test many kinetic energies due to practical limitations such as accelerator beam time availability and the scarcity of funds to pay for accelerator testing. As a result of these limitations, computational methods will continue to play a prominent role in characterizing space radiation shielding effectiveness, especially for very low and very high kinetic energies. Measurements of selected kinetic energies should be performed as validation of the results generated using computational methods, ensuring that astronaut waste stream variability does not result in unacceptably large variation in shielding characteristics among the tiles.

This work was supported by the NASA Advanced Exploration Systems (AES) program RadWorks project. We thank Bobbie Gail Swan and Catherine Mcleod, AES RadWorks Project Management, for supporting this study. We also thank Michael Sivertz at the NSRL for his work and advice during beam line data acquisition. Finally, we thank J. Kenneth Shultis for reviewing the draft paper and providing helpful comments. Funding from the Kansas State University Office of Engineering Research and Graduate Programs and Department of Mechanical and $\mathrm{Nu}$ - clear Engineering supported data analysis, paper preparation, and conference travel.

\section{References}

[1] M. Shavers, N. Zapp, R. Barber, J. Wilson, G. Qualls, L. Toupes, S. Ramsey, V. Vinci, G. Smith, F. Cucinotta, Adv Space Res 34(6) 1333-1337 (2004)

[2] R. Singleterry, K. Bollweg, T. Martin, S. Westover, R. Battiston, W. Burger, R. Meinke, NASA/TP-2015218689 (2015)

[3] A. Emmanuel, J. Raghavan, Adv Space Res 56, 12881296 (2015)

[4] S. Walker, M. Clowdsley, H. Abston, A. Gallegos, M. Simon, 43rd ICES (2013)

[5] J. Broyan, M. Ewert, P. Fink, AIAA SPACE 2014 (2014)

[6] R. Zhang, P. Taddei, M. Fitzek, W. Newhauser, Phys Med Biol 55(9) 2481-2493 (2010)

[7] R. English, R. Benson, J. Bailey, C. Barnes, NASA TN D-7080 (1973)

[8] J. Simpson, Annu Rev Nucl Part S 33 323-382 (1983)

[9] F. Badavi, J. Wilson, A. Hunter, NASA/TP-2005213941 (2005)

[10] J. King, J Spacecr Rockets 11, 401-408 (1974)

[11] A. Bahadori, M. Van Baalen, M. Shavers, C. Dodge, E. Semones, W. Bolch, Phys Med Biol 56(6) (2011)

[12] A. Bahadori, T. Sato, T. Slaba, M. Shavers, E. Semones, M. Van Baalen, W. Bolch, Phys Med Biol 58(20) 7183-7207 (2013)

[13] J. Wilson, F. Cucinotta, J. Shinn, L. Simonsen, R. Dubey, W. Jordan, T. Jones, C. Chang, M. Kim, Radiat Meas 30, 361-382 (1999)

[14] G. Badhwar, F. Cucinotta, Radiat Res 153, 1-8 (2000)

[15] S. Guetersloh, C. Zeitlin, L. Heilbronn, J. Miller, T. Komiyama, A. Fukumura, Y. Iwata, T. Murakami, M. Bhattacharya, Nucl Instrum Methods Phys Res B 252 319-332 (2006)

[16] U.S. Department of Energy, NSRL User Guide Part III. Technical Data, https://www.bnl.gov/nsrl/userguide/bragg-curvesand-peaks.php

[17] J. Ziegler, SRIM-2013 Software, http://www.srim.org/

[18] P. O’Neill, IEEE Trans Nucl Sci 57(6) 3148-3153 (2010)

[19] R. Singleterry, S. Blattnig, M. Clowdsley, G. Qualls, C. Sandridge, L. Simonsen, T. Slaba, S. Walker, F. Badavi, J. Spangler, A. Aumann, E. Zapp, R. Rutledge, K. Lee, R. Norman, J. Norbury, Acta Astronaut 68(7-8) 1086-1097 (2011)

[20] R. Kramer, H. Khoury, J. Vieira, E. Loureiro, V. Lima, F. Lima, G. Hoff, Phys Med Biol 49, 5203-5216 (2004)

[21] ICRP, Ann ICRP 21(1-3) 1-201 (1990)

[22] NCRP, NCRP Report No. 132 (2000) 